Supporting Information

\title{
A Label-Free Electrochemical Aptasensor Based on Stone-Like Gold Nanoparticles for Ultrasensitive Detection of Tetracycline
}

\author{
Beibei Xie, Haibo Peng, Ruixue Zhang, Chenhui Wang*, Yun $\mathrm{He}^{*}$
}

\section{Material}

The sequences of the kanamycin monosulfate (KM) aptamer with the following sequence: 5'-NH $2-\left(\mathrm{CH}_{2}\right)_{6}$-TCTGGGGGTTGAGGCTAAGCCGACAG-3' were purchased from Shanghai Sangon Biological Engineering Technology \& Services Co., Ltd (Shanghai, China). Tetracycline hydrochloride (TET), doxycycline hydrochloride (DOX), oxytetracycline hydrochloride (OTC), diclofenac sodium salt (DCF), KM, potassium ferricyanide $\left(\mathrm{K}_{3}\left[\mathrm{Fe}(\mathrm{CN})_{6}\right]\right)$, ferrocyanide $\left(\mathrm{K}_{4}\left[\mathrm{Fe}(\mathrm{CN})_{6}\right]\right)$, 1-Ethyl-3-(3-dimethylaminopropyl) carbodiimide hydrochloride (EDC) and Nhydroxysuccinimide (NHS), dopamine, $\mathrm{HAuCl}_{4} \cdot 4 \mathrm{H}_{2} \mathrm{O}$ and ethanolamine were purchased from Sigma-Aldrich. Graphite flakes $(99.9995 \%$ ) with a lateral width of 2-15 $\mu \mathrm{m}$ were purchased from Alfa Aesar (USA). They were all analytical grade reagents and directly used without further purification. Pure water with a resistivity of $18.2 \mathrm{M} \Omega \mathrm{cm}$ at $25^{\circ} \mathrm{C}$ was used in all experiments.

\section{Characterization}

X-ray photoelectron spectroscopy (XPS) was taken on a Phi 5300 ESCA system (PerkinElmer, U.S.A) with the $\mathrm{Mg}(\mathrm{K} \alpha)$ radiation (X-ray energy $1253.6 \mathrm{eV})$. X-ray diffraction (XRD) patterns were collected through a D8 Advance diffractometer (Bruker, Germany) with the $\mathrm{Cu} \mathrm{K} \alpha$ target $(\lambda=1.54184 \AA)$. Nitrogen $\left(\mathrm{N}_{2}\right)$ adsorption-desorption isotherms were recorded with an ASAP 2020 HD88 instrument (Micromeritics, USA). The specific surface area (SSA) was calculated using the Brunauer-Emmett-Teller (BET) method and a relative pressure range of $\mathrm{p} / \mathrm{p}_{0}$ from 0.1 to 0.3 was used for multipoint BET calculations. The pore size distribution was obtained by calculating the adsorption branch with a density functional theory (DFT) method. Scanning electron microscopy (SEM) observation was carried out using a SU-8010 (Hitachi, Japan) instrument with an operating voltage of $5 \mathrm{kV}$. The elemental composition was obtained from high resolution energy dispersive X-ray spectrometer (Bruker, Germany) attached to SEM. Transmission electron microscopy (TEM) images were obtained through a JEM-1011 TEM 
(JEOL, Japan) under an accelerating voltage of $100 \mathrm{kV}$. Raman spectra were obtained using a LabRAM HR 800 system (Horiba JY, France) with a laser of $633 \mathrm{~nm}$. Thermogravimetric analysis (TGA) was carried out from 25 to $800^{\circ} \mathrm{C}$ in air at a heating rate of $10^{\circ} \mathrm{C} / \mathrm{min}$ with a TGA-50 thermal analyzer (SHI-MADZU, Japan).

\section{Preparation and determination of milk samples}

The milk samples were bought from a supermarket in China. The milk was first diluted with PBS at the ratio of 1:10 and centrifuged for $30 \mathrm{~min}$ to obtain the intermediate layer milk serum without fat and casein. The milk serum sample was tested by the thigh performance liquid chromatography in advance to ensure that it does not contain TET. Then TET was added into the collected TET-free serum to a final concentration of $5 \times 10^{-12} \mathrm{M}, 5 \times 10^{-13} \mathrm{M}$ and $5 \times 10^{-14} \mathrm{M}$.

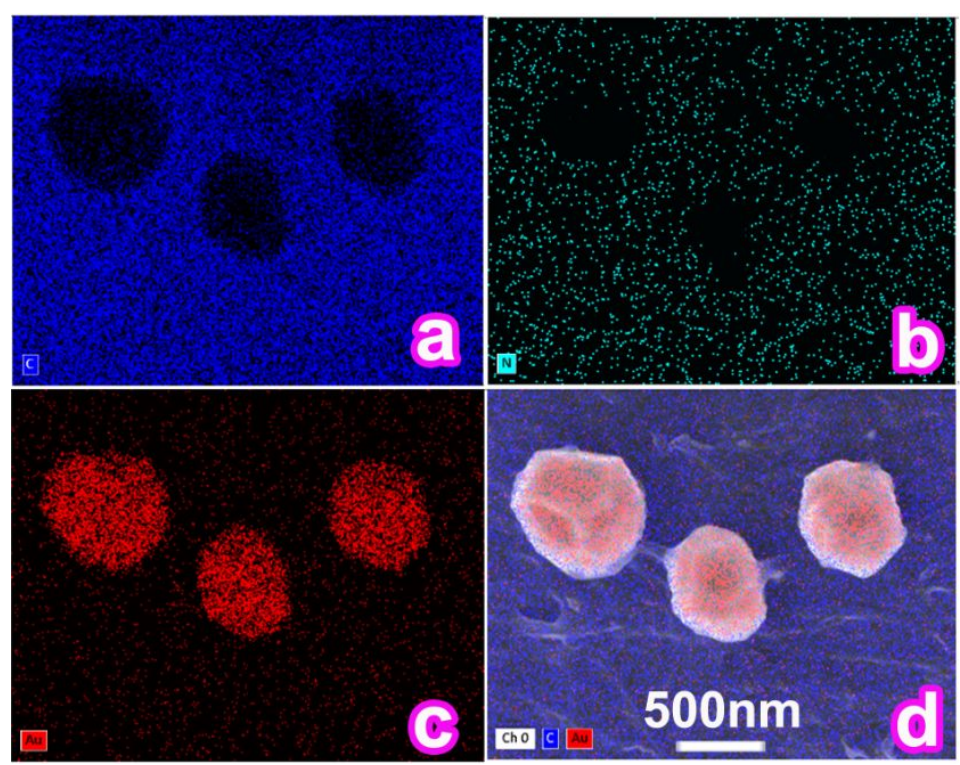

Figure S1. SEM elemental mapping images of a) C, b) N, c) Au element and d) the merged image of NCA@SL-AuNPs. 


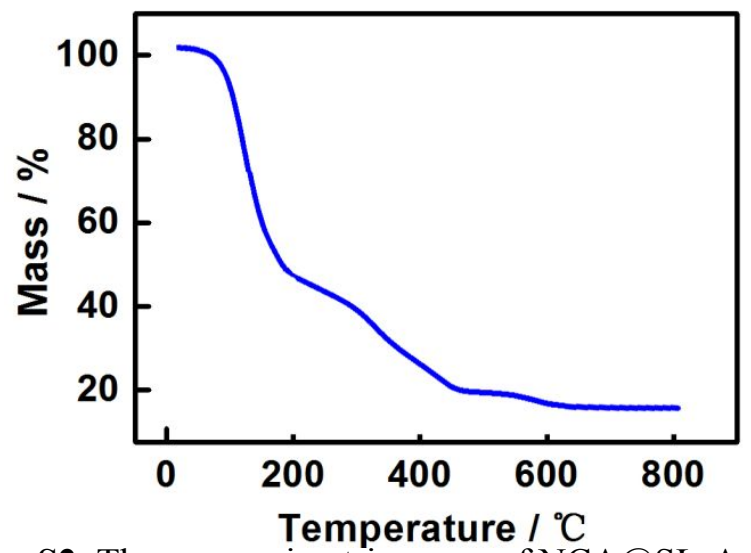

Figure S2. Thermogravimetric curve of NCA@SL-AuNPs.

Table S1. The atom ratio of NCA@SL-AuNPs.

\begin{tabular}{cc} 
Content & NCA@SL-AuNPs \\
\hline $\mathrm{N}$ (at.\%) & 4.63 \\
$\mathrm{Au}$ (at.\%) & 0.09 \\
$\mathrm{~N} / \mathrm{Au}$ (at./at.) & 51.4 \\
\hline
\end{tabular}

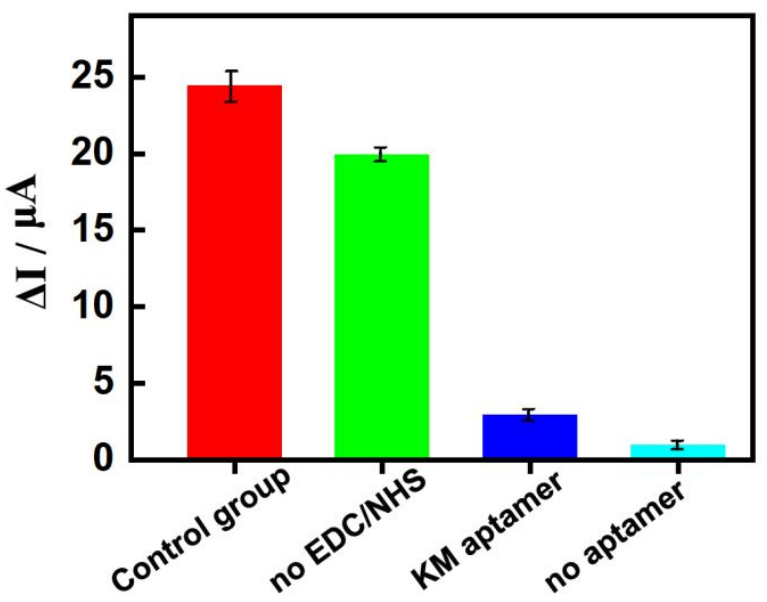

Figure S3. The effect of EDC/NHS coupling agent, with or without KM aptamer on the detection of TET. 
Table 2. The comparison between NCA@SL-AuNPs and recently reported aptasensor for TET detection.

\begin{tabular}{|c|c|c|c|}
\hline Materials & $\begin{array}{l}\text { Linear range } \\
\qquad(\mu \mathrm{M})\end{array}$ & $\begin{array}{l}\text { Detection Limit } \\
\qquad(\mu \mathrm{M})\end{array}$ & Ref. \\
\hline M-shape of aptamer/SPGEs & $1.25^{*} 10^{-3} \sim 3.5$ & $4.5^{*} 10^{-4}$ & [1] \\
\hline $\mathrm{rGO}-\mathrm{Fe}_{3} \mathrm{O}_{4} / \mathrm{SPCE}$ & $1 \sim 5000$ & $6 * 10^{-4}$ & [2] \\
\hline $\begin{array}{c}\text { CS-PB-CR/MWCNTs- } \\
\text { CS/GCE }\end{array}$ & $10^{-4} \sim 10^{3}$ & $5.6^{*} 10^{-6}$ & [3] \\
\hline AuNPs/PB-CS-GA/GCE & $0.01 \sim 10$ & $3.2 * 10^{-4}$ & {$[4]$} \\
\hline $\begin{array}{l}\text { cocktail modified gold screen- } \\
\text { printed electrodes }\end{array}$ & $2.25^{*} 10^{-5} \sim 2.25$ & $1.6 * 10^{-5}$ & [5] \\
\hline $\begin{array}{c}\text { triple helix } \\
\text { aptamer probe }\end{array}$ & $2 * 10^{-4} \sim 0.1 \mathrm{nM}$ & $1.3 * 10^{-4}$ & [6] \\
\hline MWCNTs/GCE & $0.01 \sim 10$ & $5 * 10^{-3}$ & [7] \\
\hline UV-DNA/GCE & $0.3 \sim 90$ & 0.27 & {$[8]$} \\
\hline aptamer/GE & $0.01 \sim 10$ & 0.01 & [9] \\
\hline $\mathrm{OA} / \mathrm{CPE}$ & $10^{-6} \sim 0.1$ & $5 * 10^{-7}$ & \multirow{2}{*}[10]{} \\
\hline $\mathrm{OA} / \mathrm{Fe}_{3} \mathrm{O}_{4} / \mathrm{MBCPE}$ & $10^{-6} \sim 10$ & $3.8^{*} 10^{-9}$ & \\
\hline $\mathrm{GO} / \mathrm{GCE}$ & $10^{-7} \sim 10$ & $2.9 * 10^{-8}$ & [11] \\
\hline aptamer/GE & $2.25^{*} 10^{-2} \sim 6.75$ & $2.5^{*} 10^{-3}$ & [12] \\
\hline aptamer/GE & $1.25 * 10^{-3} \sim 11.25$ & $2.5^{*} 10^{-4}$ & [13] \\
\hline nano ATO-CS/IDAMs & $0.025 \sim 2250$ & $6.75^{*} 10^{-3}$ & [14] \\
\hline $\begin{array}{c}\mathrm{MB} / \mathrm{anti}-\mathrm{TC} \\
\text { aptamer/DpAu/GCE }\end{array}$ & $10^{-4} \sim 10^{-3}$ & $4.2 * 10^{-5}$ & [15] \\
\hline NCA@SL-AuNPs & $10^{-9} \sim 10^{-5}$ & $3.2 * 10^{-10}$ & This work \\
\hline
\end{tabular}

M-shape of aptamer/SPGEs: M-shape of aptamer modified screen-printed gold electrodes;

rGO-Fe $\mathrm{O}_{4} / \mathrm{SPCE}$ : reduced $\mathrm{GO}$ and magnetite $\mathrm{Fe}_{3} \mathrm{O}_{4}$ nanoparticles modified screen-printed carbon electrodes;

CS-PB-CR/MWCNTs-CS/GCE: multi-walled carbon nanotubes-chitosan and chitosan-prussian blue-graphene nanoparticals modified glass carbon electrode;

AuNPs/PB-CS-GA/GCE: prussian bluechitosan-glutaraldehyde doped gold nanoparticals modified glass carbon electrode; UVDNA/GCE: UV irradiated DNA film modified glassy carbon electrode;

aptamer/GE: aptamer modified gold electrode; OA/CPE: oleic acid modified carbon paste electrode;

$\mathrm{OA} / \mathrm{Fe}_{3} \mathrm{O}_{4} / \mathrm{MBCPE}$ : magnetite $\mathrm{Fe}_{3} \mathrm{O}_{4}$ nanoparticles and oleic acid modified carbon paste electrode; Ab-MNPs-CS/GE: monoclonal antibody and $\mathrm{Fe}_{3} \mathrm{O}_{4}$ nanoparticles doped chitosan modified gold electrode; $\mathrm{GO} / \mathrm{GCE}$ : graphene oxide modified glassy carbon electrode;

Nano ATO-CS/IDAMs: antimony tin oxide nanoparticle-chitosan modified interdigitated array microelectrodes;

$\mathrm{MB} /$ anti-TC aptamer/DpAu/GCE: Methylene blue coated anti-TC aptamer modified glassy carbon electrode with electrodeposition gold nanoparticals; 
Table S3. Recoveries of TET from spiked milk samples $(n=3)$.

\begin{tabular}{ccccc}
\hline Sample & $\begin{array}{c}\text { Standard } \\
\text { concentration }(\mathrm{M})\end{array}$ & $\begin{array}{c}\text { Detection } \\
\text { concentration }(\mathrm{M})\end{array}$ & Recovery (\%) & $\begin{array}{c}\text { relative standard } \\
\text { deviation (\%) }\end{array}$ \\
\hline 1 & $5 \times 10^{-12}$ & $4.96 \times 10^{-12}$ & 99.2 & 4.82 \\
2 & $5 \times 10^{-13}$ & $4.93 \times 10^{-13}$ & 98.6 & 5.21 \\
3 & $5 \times 10^{-14}$ & $4.94 \times 10^{-14}$ & 98.8 & 4.68 \\
\hline
\end{tabular}

\section{References}

[1] Taghdisi, S. M., Danesh, N. M., Ramezani, M., Abnous, K., 2016. Biosensors and Bioelectronics 85, 509-514.

[2] Zhan, X., Hu, G., Wagberg, T., Zhan, S., Xu, H., Zhou, P. 2016. Microchimica Acta 183, 723-729.

[3] Guo, Y., Shen, G., Sun, X., Wang, X., 2014. IEEE Sensors Journal 15, 1951-1958.

[4] Shen, G., Guo, Y., Sun, X., Wang, X., 2014. Nano-Micro Letters 6, 143-152.

[5] Huang, Y., Yan, X., Zhao, L., Qi, X., Wang, S., Liang, X., 2019. Microchemical Journal 150, 104179.

[6] Wang, Y., Yao, L., Ning, G., Wu, Y., Wu, S., Mao, S., Liu, G.-Q., 2019. Biosensors and Bioelectronics 143, 111613.

[7] Niu, S., Zhao, M., Hu, L., Zhang, S., 2008. Sensor Actuat. B-Chem. 135, 200-205.

[8] Gholivand, M. B., Khani, H., 2013. Electroanalysis 25, 461-467.

[9] Kim, Y. J., Kim, Y. S., Niazi, J. H., Gu, M. B., 2010. Bioprocess and biosystems engineering, 33, 31.

[10] Jahanbani, S., Benvidi, A., 2016. Biosensors and Bioelectronics 85, 553-562.

[11] Benvidi, A., Tezerjani, M. D., Moshtaghiun, S. M., Mazloum-Ardakani, M., 2016. Microchimica Acta 183, 1797-1804.

[12] Le, T. H., Pham, V. P., La, T. H., Phan, T. B., Le, Q. H., 2016. Advances in Natural Sciences: Nanoscience and Nanotechnology 7, 015008.

[13] Chen, D., Yao, D., Xie, C., Liu, D., 2014. Food Control 42, 109-115.

[14] Xu, Q. C., Zhang, Q. Q., Sun, X., Guo, Y. M., Wang, X. Y., 2016. RSC Advances 6, 17328-17335.

[15] Guo, Y., Wang, X., Sun, X., 2015. Int. J. Electrochem. Sci 10, 3668-3679. 